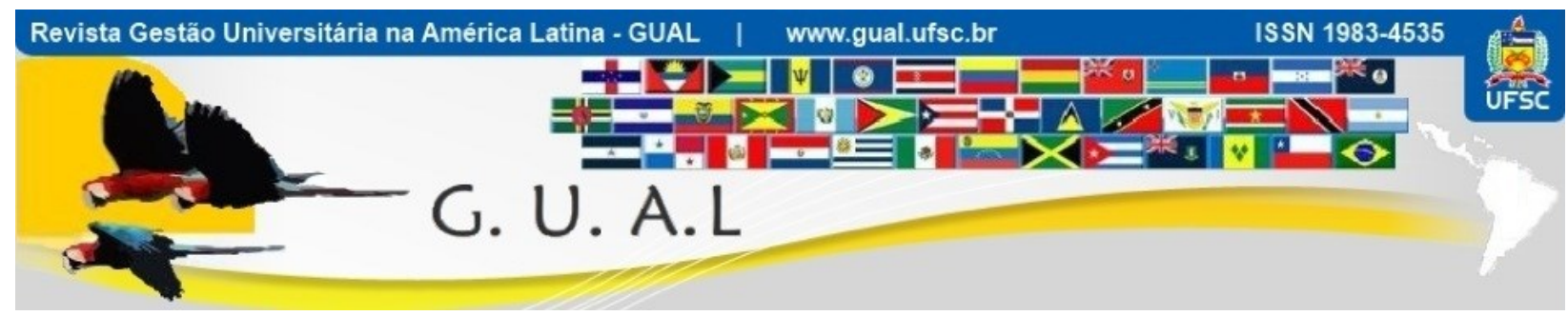

DOI: http://dx.doi.org/10.5007/1983-4535.2015v8n4p20

\title{
LAS OFICINAS DE RELACIONES INTERNACIONALES EN EL PROCESO DE INTERNACIONALIZACIÓN DE LA EDUCACIÓN SUPERIOR. UN ANÁLISIS A TRAVÉS DE VARIABLES DE GESTIÓN
}

\author{
INTERNATIONAL RELATIONS OFFICES IN THE PROCESS OF \\ INTERNATIONALIZATION OF HIGHER EDUCATION. AN ANALYSIS BY \\ MANAGEMENT VARIABLES
}

María Julieta Abba, Mestre

Universidade do Vale do Rio Dos Sinos - UNISINOS mjulieta.abba@gmail.com

Recebido em 05/dezembro/2014

Aprovado em 05/abril/2015

Sistema de Avaliação: Double Blind Review

Esta obra está sob uma Licença Creative Commons Atribuição-Uso. 


\section{LAS OFICINAS DE RELACIONES INTERNACIONALES EN EL PROCESO DE INTERNACIONALIZACIÓN DE LA EDUCACIÓN SUPERIOR. UN ANÁLISIS A TRAVÉS DE VARIABLES DE GESTIÓN \\ DOI: http://dx.doi.org/10.5007/1983-4535.2015v8n4p20}

\section{RESUMEN}

En términos históricos, se considera que la dimensión internacional de las universidades estuvo presente desde la creación de las mismas. Sin embargo, recién en las últimas dos décadas ha cobrado un nuevo rumbo en un contexto de creciente globalización, en el cual las instituciones de educación superior ya no se conciben aisladamente, sino que, por el contrario, se encuentran interconectadas entre sí superando las barreras geográficas. Por tal motivo, en los últimos años se advierte un incremento en la participación de las universidades en el proceso de Internacionalización de la Educación Superior a través de la creación de espacios institucionales dedicados a su vinculación internacional.

En este marco, el trabajo propone abordar la gestión de las Oficinas de Relaciones Internacionales de las Universidades Nacionales Argentinas, a través de un estudio de caso comparativo de dos universidades grandes (Universidad Nacional de Buenos Aires y Universidad Nacional de La Plata), dos universidades medianas (Universidad Nacional del Centro de la Provincia de Buenos Aires y Universidad Nacional de Mar del Plata) y dos universidades pequeñas (Universidad Nacional de Tres de Febrero y Universidad Nacional de San Martín). La investigación se desarrolla mediante un análisis de seis variables de gestión de la internacionalización implementadas por las Oficinas de Relaciones Internacionales y su vinculación con el tamaño y trayectoria de las instituciones donde ellas se encuentran. Entre las variables seleccionadas están la planificación estratégica, la gestión de programas y proyectos, los procesos de evaluación y monitoreo, la decisión política de las autoridades universitarias, la profesionalización y capacitación del personal que trabaja en la ORI y, finalmente, la vinculación de la investigación en los procesos de gestión.

Palabras clave: Internacionalización. Educación Superior. Oficinas de Relaciones Internacionales. Gestión. Variables.

\section{ABSTRACT}

In historical terms, it is considered that the international dimension of universities was there since the creation of these institutions. However, only in the last two decades has taken a new direction in a context of increasing globalization, in which higher education institutions are no longer conceived in isolation, but there are some cases of intitutions interconnected to each other overcoming geographical barriers. Therefore, in recent years it is noticed an increase in the participation of universities in the process of Internationalization of Higher Education through the creation of institutional spaces dedicated to its international linkages.

In this context, the work aims to address the management of International Relations Offices of the Argentine National Universities, through a comparative case study of two large universities (Universidad Nacional de Buenos Aires y Universidad Nacional de La Plata), two medians universities (Universidad Nacional del Centro de la Provincia de Buenos Aires y Universidad Nacional de Mar del Plata), and two small universities (Universidad Nacional de Tres de Febrero y Universidad Nacional de San Martín). The research was developped through an analysis of six variables of internationalization management, implemented by the International Relationship Office, and its linkage to the size and trajectory of the institutions in which they are found. The selected variables are the strategic planning; the management of programs and projects; the evaluation and monitoring processes; the political decision of the university authorities; the professionalization and training of personnel working in the office and, finally, the linking research management process.

Keywords: Internationalization. Higher Education. Offices of International Relations. Management. Variables. 


\section{INTRODUCCIÓN}

El presente trabajo forma parte de una investigación realizada en el marco de la maestría en Políticas y Administración de la Educación de la Universidad Nacional de Tres de Febrero (UNTREF), cuyo objetivo central fue analizar variables de gestión de la vinculación internacional de las Oficinas de Relaciones Internacionales (ORI's) de 6 Universidades Nacionales Argentinas que fueron seleccionadas.

Para ello, en una primera parte, se abordaran diversos aportes y reflexiones teóricas que contribuyan para la generación de una comprensión endógena de la Internacionalización de la Educación Superior. Luego, se analizará la gestión de la vinculación internacional de las universidades, haciendo hincapié en las ORI's como protagonistas del proceso de internacionalización. En tercer lugar, se realizará una descripción de las 6 variables de gestión implementadas por las ORI's, entre las que se encuentran la planificación estratégica, la gestión de programas y proyectos, los procesos de evaluación y monitoreo, la decisión política de las autoridades universitarias, la capacitación del personal que trabaja en la ORI y, finalmente, la vinculación de la investigación en los procesos de gestión. En cuarto lugar, se presentará la metodología utilizada durante el trascurso de la investigación, para luego hacer referencia al análisis comparativo de las variables de cada una de las universidades seleccionadas. Por último, se presentarán algunas reflexiones finales en torno a lo expuesto a lo largo del trabajo.

\section{LA INTERNACIONALIZACIÓN DE LA EDUCACIÓN SUPERIOR. APORTES TEÓRICOS PARA UNA COMPRENSIÓN ENDÓGENA}

El comienzo de la dimensión internacional de las universidades puede remontarse a los propios orígenes de dichas instituciones, aproximadamente durante el siglo XII y XIII, en la Europa occidental. En aquellas épocas profesores y estudiantes viajaban a otras instituciones educativas, ubicadas en localidades geográficas diferente, con el objetivo de realizar intercambios académicos y nutrirse de esas experiencias (García Guadilla, 2005). De esta manera, el comienzo de la internacionalización se localizó principalmente en Europa y luego se extendió a los Estados Unidos y Canadá.

En función de lo desarrollado en el párrafo anterior, cabría preguntarse ¿cuál es la principal diferencia de los primeros pasos de la Internacionalización de la Educación Superior $\mathrm{y}$ el proceso que vivimos actualmente en nuestras universidades? Durante el siglo XX, la 


\section{LAS OFICINAS DE RELACIONES INTERNACIONALES EN EL PROCESO DE INTERNACIONALIZACIÓN DE LA EDUCACIÓN SUPERIOR. UN ANÁLISIS A TRAVÉS DE VARIABLES DE GESTIÓN \\ DOI: http://dx.doi.org/10.5007/1983-4535.2015v8n4p20}

internacionalización fue identificada con una de sus actividades principales: la movilidad internacional. No obstante, hacia finales de ese siglo comenzó una preocupación, tanto de la academia como de las propias universidades, por un lado en abordar conceptualmente a la internacionalización, es decir, la realización de investigaciones y reflexiones en profundidad sobre la internacionalización como objeto de estudio específico dentro del campo de la educación superior; y por el otro, un interés creciente en el financiamiento y la planificación de las actividades de vinculación internacional de las casas de altos estudios.

Vale destacar que este proceso no fue homogéneo en todo el mundo, ya que el mayor desarrollo se localizó en el hemisferio norte, posicionando a unas pocas regiones en la vanguardia sobre la temática. Dan cuenta de ello, un sinnúmero de trabajos realizados durante los '90, principalmente por autores canadienses, europeos y estadounidenses. Algunos de ellos, fueron Knight (1994) y de Wit (1995), pioneros en realizar investigaciones que analizaban la Internacionalización de la Educación Superior y la definían conceptualmente. El primero de ellos, señalaba en su escrito que la internacionalización consistía en "el proceso de integración de una dimensión internacional/intercultural en las funciones de la enseñanza, la investigación y el servicio de la institución” (Knight, 1994: 2). Esta definición es la más utilizada en los ámbitos académicos y gubernamentales, ya que presenta a la internacionalización como un proceso intercultural, que no solo se orienta a los estados como tal, sino también a diferentes grupos culturales de un país. Asimismo, comprende las diversas funciones de la universidad en su totalidad lo que permite analizar a la internacionalización de manera transversal a sus diversas funciones. En un desarrollo teórico posterior, Knight (2005) destacó que la definición que elaboró fue intencionalmente neutra y objetiva, ya que debía utilizarse para describir un fenómeno universal. En esta perspectiva señaló que "una definición tiene que ser suficientemente genérica para aplicarla a muchos países con culturas y sistemas educativos diferentes" (Knight, 2005:54).

A comienzos del siglo XXI, este desarrollo desigual presentó algunos cambios, acompañados de un proceso que comienzo a gestarse en estos años y que encuentra eco en instituciones como el Mercado Común del Sur (MERCOSUR), la Unión de Naciones Sudamericanas (UNASUR), la Alternativa Bolivariana para los Pueblos de Nuestra América (ALBA) y la Comunidad de Estados Latinoamericanos y Caribeños (CELAC). En este contexto se siembra la posibilidad de introducir en las universidades una concepción internacional que comienza a cuestionar y contrarrestar el desarrollo de una 


\section{LAS OFICINAS DE RELACIONES INTERNACIONALES EN EL PROCESO DE INTERNACIONALIZACIÓN DE LA EDUCACIÓN SUPERIOR. UN ANÁLISIS A TRAVÉS DE VARIABLES DE GESTIÓN \\ DOI: http://dx.doi.org/10.5007/1983-4535.2015v8n4p20}

internacionalización exógena, fundada en valores netamente mercantiles, para transitar un camino diferente sustentado bajo los cimientos de la solidaridad, la cooperación, la horizontalidad, y la búsqueda colectiva de soluciones a los problemas que aquejan a la región, en este caso en materia de educación superior. Estos principios fueron incorporados en la Declaración de la Conferencia Regional de Educación Superior de América Latina y el Caribe (CRESALC), celebrada del 4 al 6 de junio de 2008, en la ciudad de Cartagena de Indias, Colombia. En dicho documento se resaltó la importancia de construir un espacio latinoamericano y caribeño de educación superior que contribuya a la integración regional mediante el trabajo mancomunado de los siguientes aspectos:

“...la profundización de su dimensión cultural; el desarrollo de fortalezas académicas
que consoliden las perspectivas regionales ante los más acuciantes problemas
mundiales; el aprovechamiento de los recursos humanos para crear sinergias en
escala regional; la superación de brechas en la disponibilidad de conocimientos y
capacidades profesionales y técnicas; la consideración del saber desde el prisma del
bienestar colectivo; y la creación de competencias para la conexión orgánica entre el
conocimiento académico, el mundo de la producción, el trabajo y la vida social, con
actitud humanista y responsabilidad intelectual” (CRESALC, 2008),

En este sentido, a se puede considerar que en América Latina y el Caribe comienza un proceso más activo, dando cuenta de una "nueva fase de proyección y de internacionalización de la Educación Superior en nuestros países" (Didriksson, 2008:15). Ello también repercutió en el incremento de trabajos realizados por de autores latinoamericano y caribeños sobre la internacionalización de las universidades. Algunos ejemplos provienen principalmente de México (Gacel Ávila, 2000, 2004, 2005, 2009; Didou Aupetit, 2007), Colombia (Jaramillo, 2003), Cuba (Domínguez Menéndez, 2003; López Segrera 2007a, 2007b, 2012), Venezuela (García Guadilla, 2005, 2010), Chile (Brunner, 2005), Brasil (Pereira Laus y Morosini, 2005; Morosini 2006; Leite y Genro, 2012) y Argentina (Beneitone, 2008; Pini, 2008; Theiler, 2005; Siufi, 2009; Fernández Lamarra, 2010). Cabe destacar que la mayoría de estos autores provienen del ámbito de la gestión de las universidades, como así también su procedencia tiene anclaje en la investigación y la docencia.

Este análisis sobre el advenimiento de otro tipo de internacionalización a partir de la primera década del siglo XXI, fue planteado en trabajos anteriores (Taborga et al, 2013; Abba, 2014) en los cuales se destacó un debate sobre dos tipos ${ }^{1}$ de internacionalización

\footnotetext{
${ }^{1}$ Se presentan dos visiones como tipos ideales, según la concepción weberiana. No obstante, en la práctica se pueden encontrar ambos modelos combinados en uno o más casos.
} 


\section{LAS OFICINAS DE RELACIONES INTERNACIONALES EN EL PROCESO DE INTERNACIONALIZACIÓN DE LA EDUCACIÓN SUPERIOR. UN ANÁLISIS A TRAVÉS DE VARIABLES DE GESTIÓN \\ DOI: http://dx.doi.org/10.5007/1983-4535.2015v8n4p20}

universitaria. Por un lado una exógena, que se sustenta en una concepción de internacionalización guiada por una lógica mercantil y orientada principalmente por las agendas que fijan los organismos internacionales, y por el otro, un tipo de internacionalización endógena, guiada por políticas nacionales y regionales que proponen una visión autónoma y estratégica de internacionalización. Bajo esta clasificación, el primer modelo responde a intereses y políticas que, en la mayoría de los casos, son ajenas a la realidad de las propias universidades de América Latina y el Caribe, mientras que, bajo el segundo modelo, el de la internacionalización endógena, se busca una contextualización de nuestras realidades, únicas y complejas, para luego enriquecer el conocimiento a partir de una mirada desde el sur. En consonancia con esta reflexión, podemos encontrar dos trabajos, recientemente publicados en Argentina, que contribuyen a la profundización de este debate. Uno de ellos es el libro coordinado Rinesi (2013) titulado "Ahora es cuando. Internacionalización e integración regional universitaria en América Latina”, y el otro es el libro realizado en abril de 2014 por la Comisión de Asuntos Internacionales de la Red de Universidades Nacionales del Conurbano Bonaerense (RUNCOB), cuyo título es "Desde el sur: miradas sobre la internacionalización".

Por ello, una de las definiciones de Internacionalización de la Educación Superior que se utilizó como referencia en la investigación, fue la de Beneitone (2008), quien considera que la internacionalización es una respuesta del mundo académico frente a la globalización, en donde la universidad se apropia de los valores positivos de la globalización que trascienden la mercantilización, la competitividad y las desigualdades, perfilándose en favor de la construcción de una sociedad del conocimiento "apoyada por una cooperación solidaria, horizontal, propiciando pertinencia, calidad, equidad y accesibilidad" (Beneitone, 2008: 12).

\section{LA GESTIÓN DE LA VINCULACIÓN INTERNACIONAL DE LAS UNIVERSIDADES. LAS ORI'S COMO PROTAGONISTAS DEL PROCESO DE INTERNACIONALIZACIÓN}

Como se mencionó anteriormente, el interés por parte de las universidades sobre la internacionalización universitaria tuvo un fuerte crecimiento hacia fines del siglo XX y la primera década del siglo XXI. Este proceso trajo aparejado que dichas instituciones crearan y expandieran áreas específicas dedicadas a las relaciones internacionales universitarias. Estos espacios reciben diversas denominaciones sujetas a la importancia que adquiera la política de 


\section{LAS OFICINAS DE RELACIONES INTERNACIONALES EN EL PROCESO DE INTERNACIONALIZACIÓN DE LA EDUCACIÓN SUPERIOR. UN ANÁLISIS A TRAVÉS DE VARIABLES DE GESTIÓN \\ DOI: http://dx.doi.org/10.5007/1983-4535.2015v8n4p20}

internacionalización en la universidad, que también determinará el lugar que ocupe en el organigrama institucional. Sin embargo, más allá de las diversas denominaciones que adquiera este espacio, generalmente cuando se hace referencia al mismo se lo denomina Oficina de Relaciones Internacionales (ORI). Gacel Ávila (2009) en el material teórico "Casos prácticos para la gestión de la internacionalización de las universidades", señala que "las instituciones de educación superior desarrollan (...) distintos modelos de estructuras internas que ofrecen servicios de apoyo a las actividades internacionales denominados genéricamente ORI's" (Gacel Ávila, 2009:5).

La autora considera que este nombre "destaca la importancia de la internacionalización como un objetivo y un proceso (...) hacia el interior de la universidad [que] implica priorizar actitudes y planteamientos de colaboración" (Gacel Ávila, 2009:1314). Asimismo, destaca que una ORI va más allá de las meras relaciones internacionales de una universidad -vinculadas con aspectos más formales y protocolares de las relaciones interinstitucionales- y se posiciona como una herramienta que puede potenciar el vínculo internacional de la institución, como así también como un instrumento para consolidar un modelo de internacionalización a través de la transversalidad de este proceso a todos los actores, unidades académicas y secretarías de la universidad. Por ello, para dar un mensaje a la comunidad académica sobre el papel institucional de la dimensión internacional, es necesaria una denominación adecuada para el espacio de vinculación internacional.

Siguiendo con el análisis de Gacel Ávila (2009), las principales funciones y características de la ORI pueden ser las siguientes: promover, coordinar y evaluar políticas institucionales de cooperación internacional; representar a la institución frente a organismos nacionales e internacionales; ampliar la presencia internacional de la universidad; diseñar estrategias de promoción y difusión de las actividades de internacionalización de la universidad y oportunidades de cooperación internacional; gestionar y administrar recursos externos; fomentar y administrar programas institucionales para movilidad de estudiantes,

\footnotetext{
2 Publicación realizada como producto del proyecto Safiro II (Self-Financing Alternatives for International Relation Offieces) basado en una iniciativa interregional entre la Unión Europea y América Latina, cuyo objetivo es "contribuir a la calidad del proceso de internacionalización de las universidades y al fortalecimiento de la cooperación birregional América Latina-unión Europea a través del intercambio de buenas prácticas en gestión de la internacionalización, en especial en las áreas seleccionadas de Modelos de ORI, Movilidad, Marketing y Calidad". Para mayor información: http://www.safironetwork.org/ (Consultada en Septiembre de 2013).
} 


\section{LAS OFICINAS DE RELACIONES INTERNACIONALES EN EL PROCESO DE INTERNACIONALIZACIÓN DE LA EDUCACIÓN SUPERIOR. UN ANÁLISIS A TRAVÉS DE VARIABLES DE GESTIÓN \\ DOI: http://dx.doi.org/10.5007/1983-4535.2015v8n4p20}

gestores y profesores; fomentar el establecimiento de programas académicos interinstitucionales con instituciones nacionales e internacionales, entre otras.

\section{VARIABLES IDENTIFICADAS EN LA GESTIÓN DE LAS ORIS}

A partir del análisis conceptual y de la recopilación de material empírico, se pudo constatar que la sola existencia de un espacio dedicado a la vinculación internacional, no garantiza el desarrollo de la internacionalización de una universidad. Para alcanzar dicho objetivo, se procura tener una visión más general del proceso de gestión, que comprenda las diversas dimensiones de este proceso atravesadas por las siguientes variables seleccionadas: planificación estratégica; gestión de programas y proyectos; procesos de evaluación y monitoreo; decisión política de las autoridades universitarias; la capacitación del personal que trabaja en la ORI; y finalmente, la vinculación de la investigación en los procesos de gestión. Estas variables de gestión de las ORI's fueron escogidas según la recopilación y análisis de la literatura existente sobre la temática de la gestión de las ORI's, como así también a la realización de entrevistas semi-estructuradas a informantes claves y el análisis de documentación institucional.

\subsection{PLANIFICACIÓN ESTRATÉGICA EN EL ÁMBITO DE LA GESTIÓN}

Desde la gestión, la planificación se presenta como una visión prospectiva de las instituciones, es decir, cómo se ven las mismas dentro de un periodo de tiempo estipulado, desechando la intención de predecir y apostando a la previsión de diversas situaciones, es decir, planteando una visión hacia dónde quiere ir una institución, implicando una idea de proyecto. La planificación debe tener en cuenta la naturaleza de cada institución, su historia en particular y los desafíos de su propio entorno, teniendo en cuenta que proyecto institucional y planificación estratégica son dos instrumentos de la gestión que caminan por la misma senda (Donini y Donini, 2003). De esta manera, se considera que la planificación forma parte, junto con el componente administrativo y político, de un proceso más general de gobernabilidad institucional.

\subsection{GESTIÓN DE PROGRAMAS Y PROYECTOS}

Sobre la gestión de programas y proyectos de internacionalización y cooperación internacional, Gacel Ávila (2009), considera que los mismos forman parte de las funciones y 


\section{LAS OFICINAS DE RELACIONES INTERNACIONALES EN EL PROCESO DE INTERNACIONALIZACIÓN DE LA EDUCACIÓN SUPERIOR. UN ANÁLISIS A TRAVÉS DE VARIABLES DE GESTIÓN \\ DOI: http://dx.doi.org/10.5007/1983-4535.2015v8n4p20}

servicios que brinda la ORI. Por su parte, Sebastian (2004) destaca que los programas de los que participe la institución, se pueden clasificar en dos vertientes: por un lado los que forman parte de la oferta de cooperación e internacionalización de agencias y organismos internacionales y, por otro, los programas propios de la institución sobre la cooperación e internacionalización. En el primero de los casos, la oferta de programas puede provenir de organismos internacionales, fundaciones, países extranjeros, del propio gobierno, etc. con convocatorias abiertas a través de las cuáles las instituciones universitarias pueden participar. Las convocatorias se organizan y orientan a la comunidad universitaria según las prioridades institucionales y la participación de los diversos actores. Por otro lado, también se encuentran los programas que son propios de la cooperación e internacionalización universitaria que "son los instrumentos que mejor se pueden adecuar a los objetivos institucionales y a un modelo integrado de cooperación" (Sebastian, 2004:81).

\subsection{PROCESOS DE EVALUACIÓN Y MONITOREO}

Según el aporte teórico de Nirenberg, Brawerman y Ruiz (2000) la evaluación es una actividad planificada de reflexión sobre diferentes acciones del programa o proyecto, que lleva adelante procedimientos sistemáticos de "recolección, análisis e interpretación de la información", con el objetivo de expresar análisis y declaraciones valorativas de los resultados e impactos de esos programas. También permite "formular recomendaciones para tomar decisiones que permitan ajustar la acción presente y mejorar la acción futura" (Nirenberg, Brawerman y Ruiz 2000:32).

En cuanto al ciclo de vida de un programa o proyecto, podemos encontrar el proceso evaluativo en distintos momentos. En primer lugar, en la planificación del programa, a través del análisis de la formulación del diseño y la evaluación de los documentos que fueron tenidos en cuenta para la planificación; en segundo lugar, en la ejecución del programa, haciendo hincapié en el monitoreo y la evaluación de procesos para dilucidar si la planificación está cumpliendo con un mínimo de los objetivos que fueron propuestos en un principio; y finalmente, en la etapa de la finalización del programa, se produce la evaluación final de los resultados y el impacto, donde es fundamental la utilización de indicadores.

\subsection{DECISIÓN POLÍTICA DE LAS AUTORIDADES UNIVERSITARIAS}




\section{LAS OFICINAS DE RELACIONES INTERNACIONALES EN EL PROCESO DE INTERNACIONALIZACIÓN DE LA EDUCACIÓN SUPERIOR. UN ANÁLISIS A TRAVÉS DE VARIABLES DE GESTIÓN \\ DOI: http://dx.doi.org/10.5007/1983-4535.2015v8n4p20}

El grado de compromiso de las autoridades de la universidad con la internacionalización puede verse reflejado en la importancia que se le otorga en el organigrama institucional a la dimensión internacional, como así también los recursos humanos y el financiamiento que se otorga para dicho proceso (Gacel Ávila, 2009). Este espacio, dedicado a las relaciones internacionales de la universidad, puede ocupar distintos grados jerárquicos hacia adentro de la institución. Así, por ejemplo, la ORI puede depender directamente de rectorado o vicerrectorado y ser una secretaría o tener carácter de área y encontrarse dependiendo de la secretaría de relaciones institucionales de la universidad, entre otras opciones. Por ello, su denominación puede variar de la siguiente manera:

\footnotetext{
"Oficina, unidad, coordinación, dirección general, coordinación general, vicerrectoría, vicepresidencia, centro, etcétera; de asuntos internacionales, de cooperación internacional, de relaciones internacionales, de intercambio académico, de programas y servicios internacionales, de internacionalización o de educación internacional."' (Gacel Ávila, 2009:13).
}

Según Sebastián (2004), la importancia de la denominación del espacio dedicado a la vinculación internacional radica en brindar un mensaje claro a la comunidad académica sobre el papel institucional de la dimensión internacional. Asimismo, un elemento relacionado con la posición del espacio dedicado a la vinculación internacional en la universidad, es el presupuesto. A medida que la ORI tenga una mayor jerarquización en la universidad, su presupuesto se incrementará. No obstante, el hecho de que la ORI cuente con un presupuesto propio no es un elemento determinante a la hora de realizar una política internacionalización, pero contribuye a la generación de insumos, a la elaboración de un programa integral de movilidad y la capacitación de recursos humanos.

\subsection{CAPACITACIÓN DEL PERSONAL QUE TRABAJA EN LA ORI}

Diversos autores (Gacel Ávila, 2000, 2009; Sebastian, 2004) consideran que tanto los responsables de las ORI's, como el personal que allí se desempeña, deben ser profesionales formados con experiencia en internacionalización universitaria. El argumento esgrimido radica en que si la institución no cuenta con personal con este perfil, el proceso de internacionalización será más lento, no será óptimo e incluso podría fracasar.

En este marco de capacitación de recursos humanos, en lo que respecta a la ORI, resulta de valiosa importancia el dominio de idiomas extranjeros, comunicación oral y escrita, normas protocolares, conocimientos interculturales, conocimiento de programas académicos 


\section{LAS OFICINAS DE RELACIONES INTERNACIONALES EN EL PROCESO DE INTERNACIONALIZACIÓN DE LA EDUCACIÓN SUPERIOR. UN ANÁLISIS A TRAVÉS DE VARIABLES DE GESTIÓN \\ DOI: http://dx.doi.org/10.5007/1983-4535.2015v8n4p20}

de cooperación, publicaciones en el área de la internacionalización y la cooperación universitaria, etc. (Gacel Ávila, 2009). Asimismo, para lograr una visión de la internacionalización como proceso transversal a todas las áreas y funciones de la institución, resulta importante hacer partícipes de los talleres/jornadas/capacitaciones al personal que trabaja en otras secretarías que se encuentran involucradas en el proceso de vinculación internacional de la universidad.

\subsection{VINCULACIÓN DE LA INVESTIGACIÓN EN LOS PROCESOS DE GESTIÓN}

La última variable de análisis se aboca a la investigación y a todas las acciones que ella misma comprenda para analizar la internacionalización universitaria y generar información para la toma de decisiones de la gestión institucional. Así, esta actividad se convierte en una herramienta de utilidad para establecer un diagnóstico de la situación actual de la ORI y de acuerdo con ello, proceder a la elaboración de planes estratégicos, la formulación de programas y proyectos, etc. Algunas actividades del proceso de investigación se encuentran vinculadas a la sistematización de la información de la ORI; recolección, relevamiento y procesamiento de datos; análisis de la bibliografía existente sobre la temática; recolección de información y participación de las principales reuniones y eventos académicos concernientes a la cooperación e Internacionalización de la Educación Superior, entre otras.

Para llevar a cabo estas tareas, existen diversas formas mediante las cuales se puede fortalecer el vínculo gestión-investigación. Dos de ellas pueden ser, por un lado, fomentar el vínculo de la ORI con algún grupo de investigación de la propia universidad o de otra institución, que trabaje sobre temáticas de internacionalización y cooperación universitaria; y por el otro, incorporar a los recursos humanos que trabajan en la ORI, una persona o un equipo de trabajo que se encargue de dichas actividades. Un ejemplo que se puede mencionar dentro de esta opción es la inclusión de estudiantes de grado y posgrado como auxiliares o pasantes en la ORI. Con esta incorporación, además de fomentar la formación de recursos humanos, se alienta a que los estudiantes compartan, junto con el personal de la oficina, las principales dificultades de la gestión y las soluciones para afrontarlas.

\section{METODOLOGÍA}

El diseño de investigación fue el estudio de caso comparativo denominado "en profundidad" (Sampieri et al., 2006) en el cual se abordó un análisis pormenorizado de seis 


\section{LAS OFICINAS DE RELACIONES INTERNACIONALES EN EL PROCESO DE INTERNACIONALIZACIÓN DE LA EDUCACIÓN SUPERIOR. UN ANÁLISIS A TRAVÉS DE VARIABLES DE GESTIÓN \\ DOI: http://dx.doi.org/10.5007/1983-4535.2015v8n4p20}

casos de estudio, para luego establecer tendencias generales respecto a la temática. En cuanto al enfoque, la investigación fue de tipo mixto, ya que se combinaron análisis de datos cuantitativos y cualitativos para dar conocimiento sobre un fenómeno y conducir a la solución de diversos problemas.

Respecto al campo empírico de la investigación, se seleccionaron las Oficinas de Relaciones Internacionales de 6 universidades divididas en tres grupos: universidades grandes, medianas y chicas. Del primer grupo se escogió la Universidad de Buenos Aires (UBA) y la Universidad Nacional de La Plata (UNLP), del segundo la Universidad Nacional del Centro de la Provincia de Buenos Aires (UNCPBA) y la Universidad Nacional de Mar del Plata y, del último grupo, se seleccionaron la Universidad Nacional de Tres de Febrero (UNTREF) y la Universidad Nacional de San Martín (UNSAM). Una vez definidas las unidades de análisis, se procedió al análisis de cada una de las variables en las respectivas universidades. Para medir y saber cual es el comportamiento de las variables, se definieron indicadores por cada una de ellas (figura 1), los cuales fueron construidos de acuerdo a los indicadores desarrollados por Sebastián (2004) en su Trabajo sobre "Cooperación e Internacionalización de las universidades".

\begin{tabular}{|c|c|c|c|c|c|}
\hline $\begin{array}{l}\text { Planificación } \\
\text { Estra tégica }\end{array}$ & $\begin{array}{l}\text { Gestión de } \\
\text { Programas y } \\
\text { Proyectos }\end{array}$ & $\begin{array}{l}\text { Monitoreo y } \\
\text { Evaluación }\end{array}$ & $\begin{array}{c}\text { Decisión política } \\
\text { de las a utoridades } \\
\text { univ. }\end{array}$ & $\begin{array}{l}\text { Capacita ción del } \\
\text { personalde la ORI }\end{array}$ & $\begin{array}{l}\text { Vinculación } \\
\text { gestión- } \\
\text { investiga ción }\end{array}$ \\
\hline $\begin{array}{l}\text { - Existestencia de } \\
\text { Planificación } \\
\text { Estratégica } \\
\text { - Cantidad de planes } \\
\text { estratégicos desde la } \\
\text { creación de la ORI }\end{array}$ & $\begin{array}{l}\text { - Creación de PyP } \\
\text { - Cantidad de PyP } \\
\text { generados por la } \\
\text { universidad }\end{array}$ & $\begin{array}{l}\text { - Existencia de } \\
\text { evaluación de PyP } \\
\text { - Existencia de un } \\
\text { grupo de trabajo } \\
\text { dedicado a evaluar } \\
\text { - Existencia de } \\
\text { publicaciones sobre } \\
\text { el resultado de las } \\
\text { evaluaciones } \\
\text { - Existencia de } \\
\text { monitoreo de los } \\
\text { proyectos } \\
\text { - Existencia de } \\
\text { evaluación de } \\
\text { impacto } \\
\text { - Relevamientos e } \\
\text { información } \\
\text { sistematizada }\end{array}$ & $\begin{array}{l}\text { - Existencia de una } \\
\text { ORI en la estructura } \\
\text { orgánica de la } \\
\text { institución } \\
\text { - Jerarquía de la ORI } \\
\text { en el organigrama } \\
\text { - Documentos } \\
\text { institucionales en los } \\
\text { que se contempla la } \\
\text { internacionalización }\end{array}$ & $\begin{array}{l}\text { - Cantidad de personas } \\
\text { que trabajan en la } \\
\text { ORI } \\
\text { - Existencia de } \\
\text { capacitaciones } \\
\text { - Cantidad de recursos } \\
\text { humanos enviados a } \\
\text { esas capacitaciones } \\
\text { por año } \\
\text { - Cantidad de recursos } \\
\text { humanos enviados a } \\
\text { otras universidades } \\
\text { nacionales } \\
\text { - Cantidad de recursos } \\
\text { humanos enviados a } \\
\text { universidades } \\
\text { extranjeras }\end{array}$ & $\begin{array}{l}\text { - Existencia de } \\
\text { investigadores que } \\
\text { aborden la temática } \\
\text { de la } \\
\text { internacionalización } \\
\text { - Vinculación de la } \\
\text { ORI con } \\
\text { investigadores que } \\
\text { trabajen ta temática }\end{array}$ \\
\hline
\end{tabular}

Figura 1 Conjunto de indicadores por cada variable

\section{ANÁLISIS COMPARATIVO DE LAS VARIABLES}


Para realizar el análisis comparativo de las variables se procedió a la elaboración de una escala de medición que complemente el análisis cualitativo con herramientas cuantitativas, a fin de enriquecer la interpretación sobre el análisis de la gestión de las ORI's. En este sentido, se seleccionó la escala de likert como instrumento de medición para la obtención de una puntuación de acuerdo a cada variable y a cada indicador comprendido en ella. Este proceso se plasmó en el gráfico $\mathrm{N}^{\circ} 1$, en el cual se puede observar el comportamiento de cada variable en cada universidad.

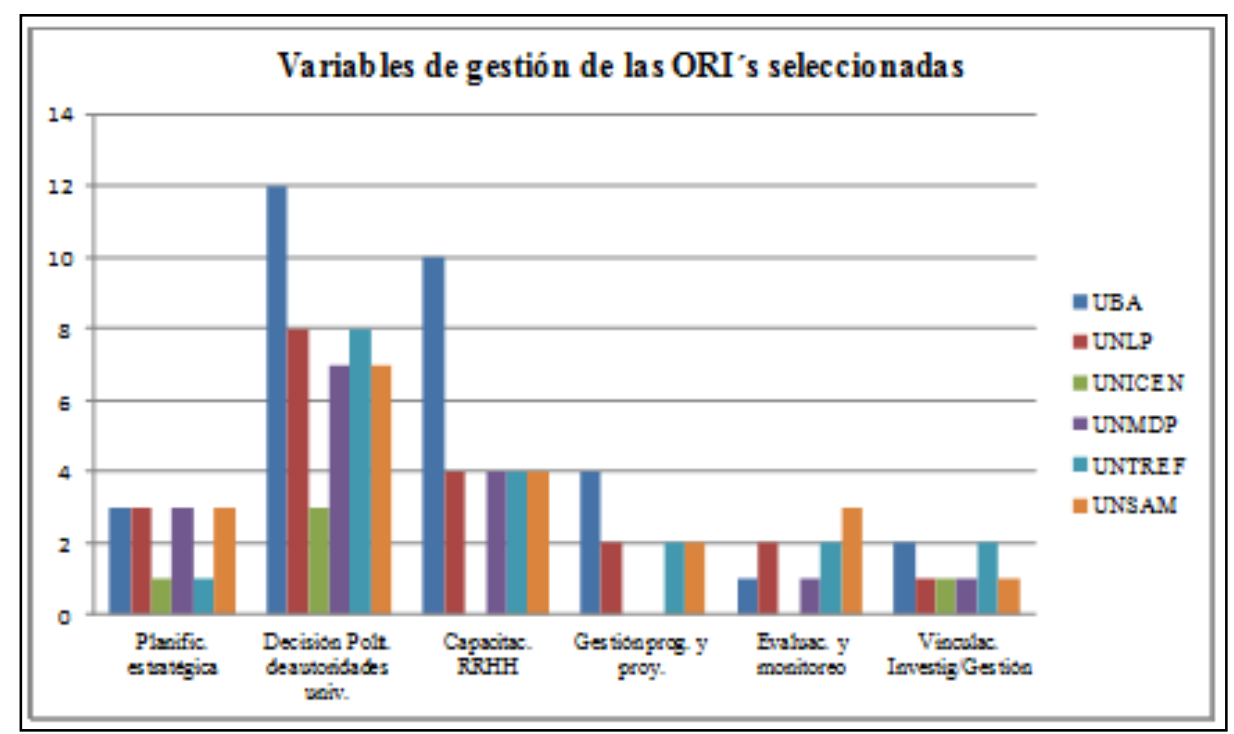

Gráfico $\mathbf{N}^{\mathbf{0}} \mathbf{1}$ Variables de gestión de las ORI's seleccionadas

Fuente: elaboración propia, en base a recopilación de información de las ORI's.

Según la información que se observa en el gráfico $\mathrm{N}^{\circ} 1$, las variables menos desarrolladas en las universidades seleccionadas son la evaluación y monitoreo, la vinculación de la investigación con la gestión y, en menor medida, la gestión de programas y proyectos propios de internacionalización y cooperación internacional. Luego, la variable que presenta un gran desarrollo en las universidades es la decisión política de autoridades universitarias. En lo que respecta a la capacitación de recursos humanos y la planificación estratégica, se encuentra un comportamiento mayoritariamente homogéneo entre las universidades, con excepción de la UBA que presenta un desarrollo notable en esta última variable.

El gráfico $\mathrm{N}^{\mathrm{o}} 2$ compara las variables en los tres grupos definidos como universidades grandes, medianas y chicas. Lo interesante de ello, radica en revelar cuál es el desarrollo de la gestión de las ORI's y el nexo de esta situación con el tamaño de la universidad. Tal vez, antes de observar el siguiente gráfico, una de las hipótesis podría ser que las variables de 
gestión de las ORI's tienen un despliegue mayor en las universidades grandes, luego en las medianas y finalmente en las universidades chicas. Sin embargo, luego de la recopilación y triangulación de los datos, los resultados plasmados en el gráfico 2 exponen una situación diferente para el grupo de las medianas y las pequeñas.

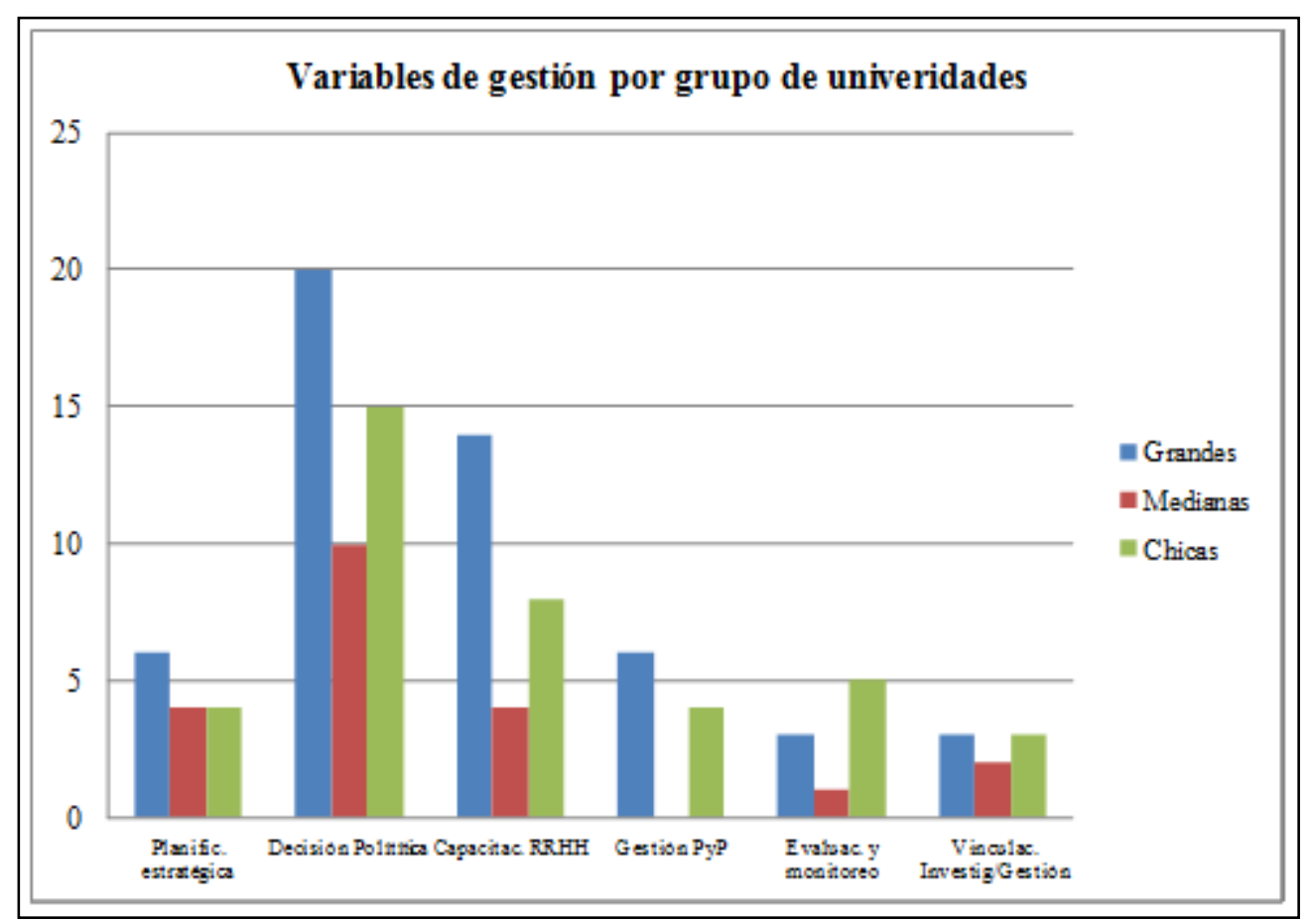

Gráfico $\mathbf{N}^{0} \mathbf{2}$ Variables de gestión por grupo de universidades

Fuente: elaboración propia, en base a recopilación de información de las ORI's.

Los resultados de las universidades grandes coincidieron con la larga trayectoria y la enorme magnitud de la UBA y la UNLP, en cuanto universidades que concentran una cantidad importante de recursos financieros, como así también de matrícula de docentes, estudiantes e investigadores. En este grupo se presentó un mayor desarrollo de la planificación estratégica, gestión de programas y proyectos, decisión política de las autoridades universitarias y capacitación de los recursos humanos. No obstante, otro aspecto que se pudo destacar en este análisis radica en que las universidades chicas en determinadas variables presentan un desarrollo igual o mayor que el grupo de universidades medianas. Tal es el caso de la capacitación de recursos humanos, la gestión de programas y proyectos, la vinculación gestión-investigación y la evaluación y monitoreo. Respecto a esta última, vale destacar que las universidades chicas presentan un desarrollo mayor, inclusive comparado con las universidades grandes. 


\section{LAS OFICINAS DE RELACIONES INTERNACIONALES EN EL PROCESO DE INTERNACIONALIZACIÓN DE LA EDUCACIÓN SUPERIOR. UN ANÁLISIS A TRAVÉS DE VARIABLES DE GESTIÓN \\ DOI: http://dx.doi.org/10.5007/1983-4535.2015v8n4p20}

Si bien la UNSAM y UNTREF cuentan con el promedio general de recursos humanos que trabajan en las ORI's de los estudios de casos seleccionados, la decisión política de sus autoridades universitarias es notable de acuerdo con desarrollo del resto de las variables. Ello sumado a dos aspectos principales, por un lado una visión estratégica de la internacionalización de estas universidades según al contexto en el que se encuentran insertas y, por el otro, la imperiosa necesidad de discutir conceptualizaciones a fines a la temáticas. Asimismo, por ser universidades de reciente creación, se insertan en un contexto de avance de la globalización y las TIC's, en el cual desde lo institucional la dinámica internacional se plantea como un aspecto indispensable para el crecimiento de las universidades, motivo por el cual dejan plasmado en sus estatutos la imperiosa necesidad de vincular internacionalmente a sus profesores, investigadores y estudiantes, como así también de integrar políticas con sus pares de la región latinoamericana. De este modo, se considera que las universidades pequeñas han intentado superar las barreras y los limitantes estructurales de su tamaño, para ir más allá de su condición y profundizar las estrategias de gestión de las ORI's acordes a su contexto local, regional e internacional.

El análisis extraído sobre las universidades medianas da cuenta que es el grupo de universidades que ha desarrollado en menor medida todas las variables, con excepción de la planificación estratégica, en la que coincide con el grupo de universidades chicas y la variable de gestión de programas y proyectos propios, la cual es inexistente tanto en la UNMDP como en la UNCPBA. De esta manera, en este caso no se encuentra una correlación entre el tamaño de las universidades medianas y el desarrollo de las variables de gestión de las ORI's.

\section{REFLEXIONES FINALES}

A través del análisis de las variables, se pudo constatar que todas ellas son herramientas importantes a incorporar en la vida de la gestión universitaria y, por tal motivo, trasversales a todas las actividades institucionales. Asimismo, estas variables son fundamentales para las ORI's ya que colaboran en la sostenibilidad de dichas instituciones frente a los desafíos que enfrentan en la labor cotidiana de la vinculación internacional.

En este sentido, uno de los principales hallazgos de la investigación, fue descubrir el potencial de las universidades chicas como instituciones que poseen un compromiso activo con la internacionalización y todo lo vinculado a ella. Resulta interesante que más allá de las dificultades que se les presentan por su condición de universidades de reciente creación, 


\section{LAS OFICINAS DE RELACIONES INTERNACIONALES EN EL PROCESO DE INTERNACIONALIZACIÓN DE LA EDUCACIÓN SUPERIOR. UN ANÁLISIS A TRAVÉS DE VARIABLES DE GESTIÓN \\ DOI: http://dx.doi.org/10.5007/1983-4535.2015v8n4p20}

dichas instituciones lograron traspasar los escollos ante la falta de financiamiento y la escasez de recursos humanos, para posicionarse, junto con las universidades grandes, en el camino de la internacionalización universitaria como elemento trasversal e integrado a las funciones sustantivas de la universidad de enseñanza, investigación y extensión.

Otro de los hallazgos que se pueden mencionar en este proceso de reflexión, radica en una de las variables, específicamente en la vinculación de la investigación en los procesos de gestión. Dicha dimensión, forma parte de una de las tres variables que presentan un desarrollo menor en las ORI's de las universidades seleccionadas. Sin embargo a la hora de analizar los indicadores comprendidos en esta variable, sobre la existencia de investigadores en la universidad que aborden la temática de la internacionalización y el vínculo de la ORI con esos investigadores, se descubrió que la totalidad de las universidades se manifestaron favorablemente ante el primer indicador, mientras que las respuestas del segundo indicador denotaron que la mayoría de las universidades no posee vínculos con esos investigadores. Asimismo, en las respuestas, una minoría planteó la incorporación reciente de estos actores para participar de las actividades de la ORI. Por ello, uno de los desafíos para la posteridad será establecer y el fortalecer el vínculo entre los investigadores y la ORI para el desarrollo de labores conjuntas.

Finalmente, como fue mencionado en la primer parte de este escrito, surge la necesidad de construir una visión crítica de la internacionalización de la educación superior, en la cual se contemplen las conceptualizaciones y teorizaciones realizadas por parte de los investigadores del sur. Es decir, se busca cimentar una visión endógena de estas temáticas, que comprenda la realidad de las universidades, las características de su propio medio y las complejidades que las envuelven.

\section{BIBLIOGRAFÍA}

Abba, J. (2014). Las Oficinas de Relaciones Internacionales en el proceso de Internacionalización de la Educación Superior. Un análisis a través de variables de gestión. Tesis de la Maestría en Políticas y Administración de la Educación de la Universidad Nacional de Tres de Febrero. Ciudad Autónoma de Buenos Aires.

Beneitone, P. (2008). Internacionalización de la Educación Superior y experiencia en torno a la convergencia. México. Universidad de Guadalajara. 


\section{LAS OFICINAS DE RELACIONES INTERNACIONALES EN EL PROCESO DE INTERNACIONALIZACIÓN DE LA EDUCACIÓN SUPERIOR. UN ANÁLISIS A TRAVÉS DE VARIABLES DE GESTIÓN \\ DOI: http://dx.doi.org/10.5007/1983-4535.2015v8n4p20}

De Wit, H. (1995). Strategies or Internationalization of Higher Education: A comparative Study of Australian, Canada, Europe and United States. Ámsterdam. Asociación Europea para la Educación Internacional.

Declaración de la Conferencia Regional de Educación Superior de América Latina y el Caribe (CRESAL) IESALC-UNESCO, celebrada del 4 al 6 de junio de 2008, en la ciudad de Cartagena de Indias. Disponible en: http://www.iesalc.unesco.org.ve/docs/wrt /declaracioncres_espanol.pdf (Consultado en Mayo de 2013).

Didriksson, A. (2008). Contexto global y regional de la educación superior en América Latina y el Caribe. En IESALC (2008). Tendencias de la Educación Superior en América Latina y el Caribe. Caracas. IESALC-UNESCO.

Donini, A. M. y Donini, A. O. (2003). La gestión universitaria en el siglo XXI. Desafíos de la sociedad del conocimiento a las políticas académicas y científicas. Documento de Trabajo $\mathrm{N}^{\circ}$ 107, Universidad de Belgrano. Disponible en:

http://www.ub.edu.ar/investigaciones/dt_nuevos/107_donini.pdf (Consultado en Abril de 2013).

Gacel Ávila, J. (2000). La Internacionalización de las universidades mexicanas. Políticas y estrategias institucionales. México. ANUIES.

Gacel Ávila, J. (2009). Modelo de Oficinas de Relaciones Internacionales, en: Casos Prácticos para la Gestión de la Internacionalización en la Universidades. Alicante, España, Red Safiro II.

García Guadilla, C. (2005). Complejidades de la globalización e internacionalización de la educación superior. Interrogantes para América Latina. Cuadernos del CENDES, año 22. $\mathrm{N}^{\circ}$ 58, Tercera Época.

Knight, J. (1994). Internationalization: Elements and Checkpoints. Monografía investigativa. Nº7. Oficina de Canadá para la Cooperación Internacional. Otawa.

Knight, J. (2005). Un modelo de internacionalización: respuestas a nuevas realidades y retos. En: De Wit, et al. (2005). Educación Superior en América Latina. La dimensión internacional. Bogotá. Banco Mundial. Mayol Ediciones.

Martínez Nogueira, R. (2000). Evaluación de la Gestión Universitaria. Informe preparado para la Comisión Nacional de Evaluación y Acreditación Universitaria (CONEAU), Buenos Aires.

Nirenberg, O., Brawerman, J. y Ruiz, V. (2000). Evaluar para la transformación. Innovaciones en la evaluación de programas y proyectos sociales. Buenos Aires, Paidós.

Sampieri R. et al. (2006). Metodología de la Investigación. Distrito Federal, México. Mcgraw-Hill. 
Sebastián, J. (2004). Cooperación e Internacionalización de la Universidades. Buenos Aires, Biblos.

Taborga, A., López, M. P. Oregione, S. y Abba, J. (2013). La Internacionalización de la Universidad en Argentina a principios del siglo XXI: desde una "orientación exógena" hacia una "orientación endógena”. En: Martínez, M. E., Piñero, F. J. y Delgado, S. A: El papel de la Universidad en el Desarrollo. Universidad Autónoma de Puebla, Universidad Nacional del Centro de la Provincia de Buenos Aires, CLACSO. 